\title{
Analysis of the $\eta^{\prime}$ photoproduction off the proton and preliminary beam asym- metry results at the GRAAL experiment
}

\author{
G. Mandaglio ${ }^{1,2,3, a}$, V. Bellini ${ }^{4,3}$, J. P. Bocquet ${ }^{5}$, M. Capogni ${ }^{6,7, b}$, F. Curciarello ${ }^{2,3}$, A. D'Angelo ${ }^{6,7}$, V. De Leo ${ }^{2,3}$, J.P. \\ Didelez $^{8}$, R. Di Salvo ${ }^{7}$, A. Fantini ${ }^{6,7}$, D. Franco $6,7, c$, G. Gervino ${ }^{9,10}$, F. Ghio ${ }^{11,12}$, G. Giardina ${ }^{2,3}$, B. Girolami ${ }^{11,12}$, \\ A.M. Lapik ${ }^{13}$, P. Levi Sandri ${ }^{14}$, A. Lleres ${ }^{5}$, F. Mammoliti ${ }^{4,3}$, M. Manganaro ${ }^{2,3, d}$, D. Moricciani ${ }^{7}$, A.N. Mushkarenkov ${ }^{13}$,', \\ V.G.Nedorezov ${ }^{13}$, D. Rebreyend ${ }^{5}$, N.V. Rudnev ${ }^{13}$, C. Schaerf 6,7 , M.L. Sperduto ${ }^{4,3}$, M.C. Sutera ${ }^{3}$, A. Turinge $^{13}$, V. \\ Vegna $^{6,7, e}$, and I. Zonta ${ }^{6,7}$
}

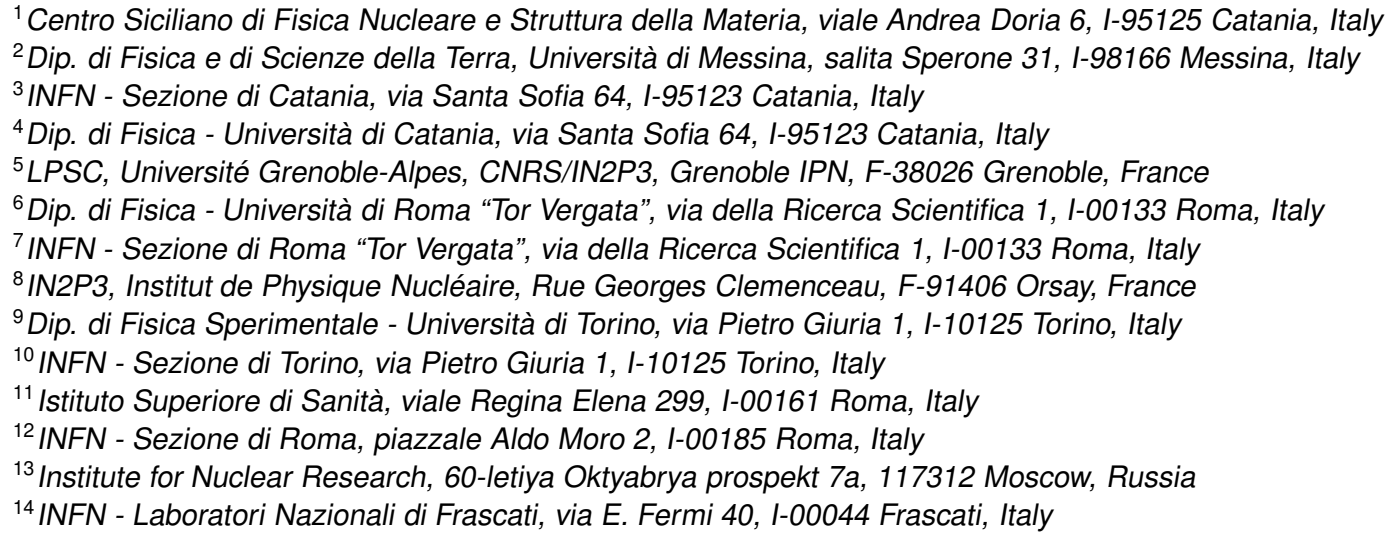

\begin{abstract}
Differential and total cross section measurements on $\eta^{\prime}$ photoproduction were published by the CLAS Collaboration (M. Dugger et al., Phys.Rev.Lett.96, 062001 (2006) and M. Williams et al., Phys.Rev.C80, 045213 (2009)) for center-of-mass energies from near the threshold up to $2.84 \mathrm{GeV}$, and by the CB-ELSATAPS Collaboration (V. Crede et al., Phys.Rev.C80, 055202 (2009)) up to $2.36 \mathrm{GeV}$ and also making a precise threshold scan of the differential cross section in the $1446-1527.4 \mathrm{MeV} \gamma$ beam energy range. However, the wide information about reaction cross sections are not sufficient to understand the role of resonances involved in the process. Different theoretical works stressed the importance to have also polarization observables in order to solve the ambiguity in the choice of the parameters used in their models.

We present the analysis of the $\eta^{\prime}$ photoproduction off the proton, identifying the meson via the $\gamma \gamma, \pi^{0} \pi^{0} \eta$, and $\pi^{+} \pi^{-} \eta$ decay modes by using the GRAAL apparatus; and we show the preliminary GRAAL results on the beam asymmetry $\Sigma$ from the threshold $(1.446 \mathrm{GeV})$ up to $1.5 \mathrm{GeV}$.
\end{abstract}

\section{Introduction}

The investigation of meson photoproduction off nucleon with polarized photons is a powerful tool to identify the broad and widely overlapped baryon resonances not easily visible with the differential cross section measurements only. Pseudo-scalar meson photoproduction is described

\footnotetext{
a e-mail: gmandaglio@unime.it

${ }^{b}$ Present affiliation: ENEA - C.R. Casaccia Istituto Nazionale di Metrologia delle Radiazioni Ionizzanti Via Anguillarese, 301 I-00123 Roma, Italy;

${ }^{c}$ Present affiliation: IPNL - 43, Bd du 11 Novembre 1918, Fr69622 Villeurbanne Cedex, France;

${ }^{\mathrm{d}}$ Present affiliation: Instituto de Astrofísica de Canarias, E-38205 La Laguna, Tenerife, Spain; Universidad de La Laguna, Dept. Astrofísica, E-38206 La Laguna, Tenerife, Spain;

e Present affiliation: Physikalisches Institut - Bonn Universität, Nussallee 12, D-53115 Bonn, Deutschland.
}

in the models by 4 complex helicity amplitudes:

$$
\begin{aligned}
\frac{d \sigma}{d \mathrm{w}} & \sim\left|H_{1}\right|^{2}+\left|H_{2}\right|^{2}+\left|H_{3}\right|^{2}+\left|H_{4}\right|^{2} \\
\Sigma & \sim \operatorname{Re}\left(H_{1} H_{4}^{*}-H_{2} H_{3}^{*}\right) \\
T & \sim \operatorname{Im}\left(H_{1} H_{2}^{*}-H_{3} H_{4}^{*}\right) \\
P & \sim \operatorname{Re}\left(H_{1} H_{3}^{*}-H_{2} H_{4}^{*}\right)
\end{aligned}
$$

Polarization observables $(\Sigma, T, P)$ are sensitive via interference between the complex helicity amplitudes, and consequently allow to reveal the small resonance contributions which remain hidden under some dominant contribution in the differential cross section [1-4]. The detailed description of the photon-nucleon interaction requires a complete data set containing, at least, eight independent observables: the cross section, the three single polarization

This is an Open Access article distributed under the terms of the Creative Commons Attribution License 4.0, which permits unrestricted use, distribution, and reproduction in any medium, provided the original work is properly cited. 
observables (beam, target and recoil nucleon) and four, appropriately chosen, double polarization observables [5]. $\eta^{\prime}$ meson, like $\eta$, has isospin equal zero and consequently $\mathrm{N} \eta^{\prime}$ final states can have only origin from intermediate nucleon states with isospin equal $1 / 2$. Therefore, $\eta^{\prime}$ photoproduction works like an isospin-filter for nucleon resonances spectrum simplifying the data interpretation and the search of excited states produced in the reaction. The $\eta^{\prime}$ meson photoproduction is also interesting because it offers the possibility to investigate the $N^{*}$ nucleon resonances, in the less explored higher $N^{*}$ mass region[6]. Recently, the CLAS experiment at Jlab and the CB-ELSA-TAPS in Bonn have produced a rich amount of cross section data on the proton [7-9] from threshold (1.447 GeV) up to 2.84 $\mathrm{GeV}$. As a consequence of this huge experimental effort, the following facts were established:

the $\eta^{\prime} \mathrm{N}$ channel couples, mainly to $\mathrm{S}_{11}(1535)$ and $\mathrm{P}_{11}(1710)$. A marginal role is played by $\mathrm{J}=3 / 2$ resonances namely $\mathrm{P}_{13}(1720)$ and $\mathrm{D}_{13}(1520)$ [7];

$\mathrm{g}_{\eta^{\prime} N N}=1.3-1.5$, a value consistent with existing theoretical estimates[7];

above $2 \mathrm{GeV}$, where the process is dominated by $\rho$ and $\omega$ exchange, the dynamics of $\eta^{\prime}$ photoproduction are similar to those of $\eta$ photoproduction [9]; flat differential cross sections are found near the photoproduction threshold [9], suggesting the s-wave dominant role in this energy range.

Different theoretical approaches developed to describe these data $[10,11]$, stressed the importance to have also polarization observables like beam asymmetry to solve the ambiguity on the parameters used in their models.

The GRAAL collaboration thanks to the stable and high polarization degree of its $\gamma$ beam and a $4 \pi$ detector, published precise single polarization observables for different meson photoproduction channels off free and quasifree proton [12-17].

In this work, we present the preliminary beam asymmetry of $\eta^{\prime}$ photoproduction off proton just over the threshold at GRAAL.

\section{GRAAL experimental set-up}

The Graal experiment worked at the European Synchrotron Radiation Facility in Grenoble (France) until the end of 2008. The experiment consisted of a polarized $\gamma$ ray beam, an unpolarized Hydrogen and Deuterium liquid target and a $4 \pi$ solid angle detector LAGRAN $\gamma \mathrm{E}$ (Large Acceptance GRaal-beam Apparatus for Nuclear $\gamma$ Experiments).

The Graal $\gamma$-ray beam is produced by the backward scattering of laser photons on the relativistic electrons of $6.03 \mathrm{GeV}$ energy circulating in the storage ring. This method was used for the first time on a storage ring for the Ladon beam at the Adone ring in Frascati [18], and it is able to produce polarized and tagged $\gamma$-ray beams with high polarization degree and good energy resolution.

The beam polarization depends on the beam energy, it is very close to the one of the laser photons (linear or circular) [19], and can be easily rotated or changed with conventional optical components changing the polariza- tion of the laser light. The correlation between photon energy and polarization is calculated with QED [20] and it is about $96 \%$ in the investigated energy over the $\eta^{\prime}$ photoproduction threshold $(1.446 \mathrm{GeV})$. With the $351 \mathrm{~nm}$ line of an Argon (Ion) Laser, the maximum $\gamma$-ray energy obtainable is about $1500 \mathrm{MeV}$ (with the multi-line far-UV is about $1550 \mathrm{MeV}$ ) and the spectrum is almost flat over the whole range. The energy resolution of the tagged beam is $16 \mathrm{MeV}$ (FWHM) over the entire spectrum. The energy of the $\gamma$-rays is provided by the tagging set-up which is located inside the ESRF shielding, attached to the ESRF vacuum system. The electrons, scattered on laser photons producing the $\gamma$-rays, lose a significant fraction of their energy escaping from the equilibrium orbit of the stored electrons and finally they are detected by the tagging system. Then, the tagging system measures the electron displacement from the equilibrium orbit. This displacement measures their energy loss in the scattering process and therefore provides a measure of the energy of the gammaray produced. The tagging system [12] consists of 10 plastic scintillators and a 128 channels Solid State Microstrip Detector with a pitch of $300 \mu \mathrm{m}$. The detector is located inside a shielding box positioned in a modified section of the ring vacuum chamber. The shielding box is positioned at $10 \mathrm{~mm}$ from the circulating electron beam.

The Graal apparatus has been described in several papers [12-17, 21, 22].

The detector covers the entire solid angle and is divided into three polar angle regions: central part $\left(25^{\circ}<\right.$ $\left.\theta \leq 155^{\circ}\right)$, forward $\left(\theta \leq 25^{\circ}\right)$, and backward $\left(\theta>155^{\circ}\right)$. In Fig. 1, we report the cross view of the central detectors and the target nose structure as drawn and used in our simulation.

A cylindrical liquid Hydrogen (or Deuterium) target is located on the beam and coaxial with it. In figure 1 a detailed view of the target nose geometry and the cell containing the liquid Hydrogen (or Deuterium) are present.

The central part, $25^{\circ}<\theta \leq 155^{\circ}$, is covered by two cylindrical multi-wire chambers (MWPC), a Barrel made of 32 plastic scintillators and a BGO crystal ball made of 480 crystals, which is well suited for the detection of $\gamma$ rays of energy below $1.5 \mathrm{GeV}$. The two MWPCs, due to their length can cover the polar angle up to $16^{\circ}$ (see Fig. 1). The chambers, the Barrel and the BGO are all coaxial with the beam and the target (see Fig. 1). The wire chambers detect and measure the positions and angles of the charged particles emitted by the target, while the scintillating Barrel measures their energy loss. The BGO ball detects charged and neutral particles measuring their deposited energy. For neutral particles it provides a measurement of their angles ( 480 crystals: 15 in the $\theta$ direction and 32 in the $\phi$ direction). The BGO and Barrel information allow to distinguish protons and charged pions by a cut on the $\Delta E_{\text {Barrel }}$ vs. $E_{B G O}$ distribution.

The solid angle cone for $\theta \leq 25^{\circ}$ is covered by two planar MWPCs, and two scintillator walls, a hodoscope and a shower detectors placed at about 3 and 3.3 metres from the target, respectively. The planar MWPCs are used to track the charged particles measuring their polar and azimuthal 


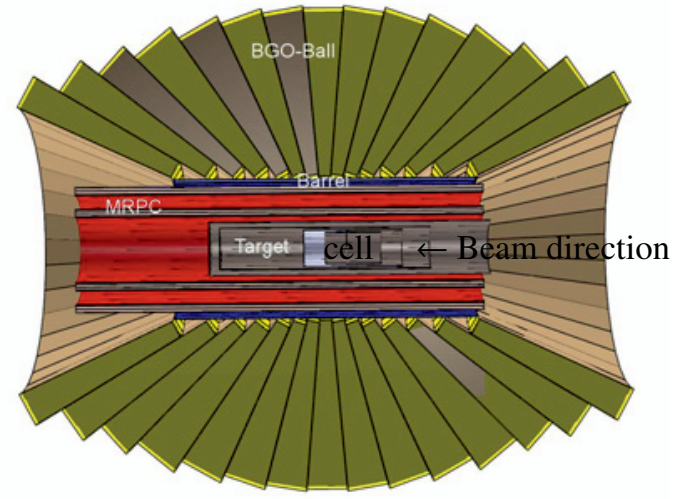

Figure 1. Cross view of 3D rendering of BGO-Ball crystal, Barrel, MRPC and target nose.

angles with a resolution of $1.5^{\circ}$ and $2^{\circ}$ (FWHM), respectively. The hodoscope wall is made of 26 horizontal and 26 vertical scintillator bars. The detector measures position, specific ionization and time of flight of charged particles. It allows to identify protons and charged pions via specific ionization and time of flight measurements. The shower wall is made of 16 vertical bars, each consisting of a sandwich of scintillators and lead to detect charged particles, $\gamma$-rays and neutrons. The TOF resolution of these scintillators is of the order of 560 ps (FWHM) for charged particles and $900 \mathrm{ps}$ for neutrons. The total thickness of the plastic scintillators is $20 \mathrm{~cm}$ and the detection efficiency is about $20 \%$ for neutrons and $95 \%$ for $\gamma$-rays. The detector is able to distinguish and identify photons and neutrons from time of flight.

Backward angles, $\theta>155^{\circ}$, are covered by two disks of plastic scintillators separated by $6 \mathrm{~mm}$ of lead to detect charged particles and gamma-rays escaping in the backward direction. It is used as veto purposes.

At the end of the beamline, two flux monitoring detectors are used.

\section{Data analysis and results}

The $\eta^{\prime}$ meson photoproduction identification via its $\gamma \gamma$, $\pi^{0} \pi^{0} \eta$ and $\pi^{+} \pi^{-} \eta$ decay modes was performed thanks to the large number of quantities measured with the LAGRAN $\gamma$ E (Large Acceptance GRaal-beam Apparatus for Nuclear $\gamma$ Experiments) detector. We were able to identify protons and charged pions everywhere in the apparatus, photons and neutrons in forward direction $\theta<$ $25^{\circ}$; to measure angles and energy (hodoscope wall) of protons, angles and energy of photons in the BGOcalorimeter, angles of charged pions with MWPCs.

The preliminary events selection was realized by using the following conditions:

i) to detect at least two neutral particles in the BGO, in order to be able to reconstruct the invariant mass, and to identify a proton in forward direction $\theta<25^{\circ}$;

ii) the energy of the beam has to overcome the $\eta^{\prime}$ photoproduction threshold $\left(E_{\gamma} \geq 1.446 \mathrm{GeV}\right)$;

iii) the measured energy of the beam $E_{\gamma}$ and polar angle of

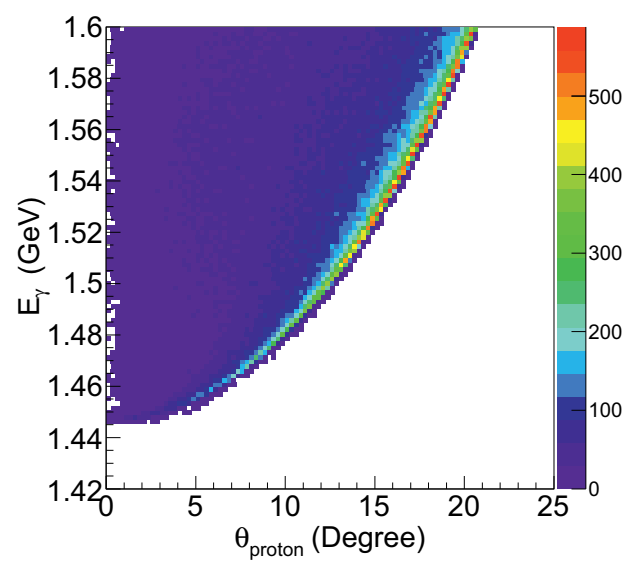

Figure 2. Energy of beam versus the proton polar angle events distribution for a simulated $\gamma p \rightarrow \eta^{\prime} p$ reaction.

the proton $\theta_{p}$ of selected events must lie in the acceptance region showed Fig. 2.

The distribution $E_{\gamma}$ vs. $\theta_{p}$ was produced with the event generator described in Ref. [23]. The distribution is due to the relation between the maximum polar angle of proton and the energy available to the reaction in the case of two body in the final state. This cut essentially rejects all events in which the mass of the particle (orthe sum of particle masses) photo-produced off the proton is lower than the $\eta^{\prime}$ mass (see Fig.3).

The study of the kinematics $\eta^{\prime}$ photo-production off proton at $50 \mathrm{MeV}$ over the threshold shows that for such energy interval the maximum values of polar angle and momentum-energy ratio of proton do not overcome 16 degrees and 0.4, respectively. Therefore, we expect to detect in our investigation a relatively slow (non relativistic) proton in the forward detectors. In such a case, the hodoscope wall is able to measure the energy of proton, from time of flight information, with a resolution of about $1 \%$, while the planar MWPCs measure the polar and azimuthal proton angles with the accuracy of $1.5^{\circ}$ and $2^{\circ}$, respectively. The resolution of proton momentum estimated with simulation in the case of $\eta^{\prime}$ photoproduction is about $2.5 \%$ for energies of the photon beam up to $1.6 \mathrm{GeV}$. The combination of the good measurement resolution of the four-momentum of proton in the forward direction and the incident photon beam, and the small gamma width of the $\eta^{\prime}$ meson mass (full width $\Gamma=0.199 \mathrm{MeV}$ ) makes the missing mass from the proton $(\gamma p \rightarrow p X)$, evaluated in the hypothesis of two body reaction in the final state, the best quantity in order to identify the events coming from the $\gamma p \rightarrow \eta^{\prime} p$ reaction. The missing mass from the detected proton was successful used also in the recent analysis of the $\omega$ meson photoproduction performed by the same collaboration (see Ref. [22]). Moreover, the analysis of simulated samples shows the possibility to measure the missing mass of proton with a narrow distribution around the $\eta^{\prime}$ mass (RMS of about $5-6 \mathrm{MeV}$ ) by using the GRAAl apparatus for energies of the beam up to $1.55 \mathrm{GeV}$. 

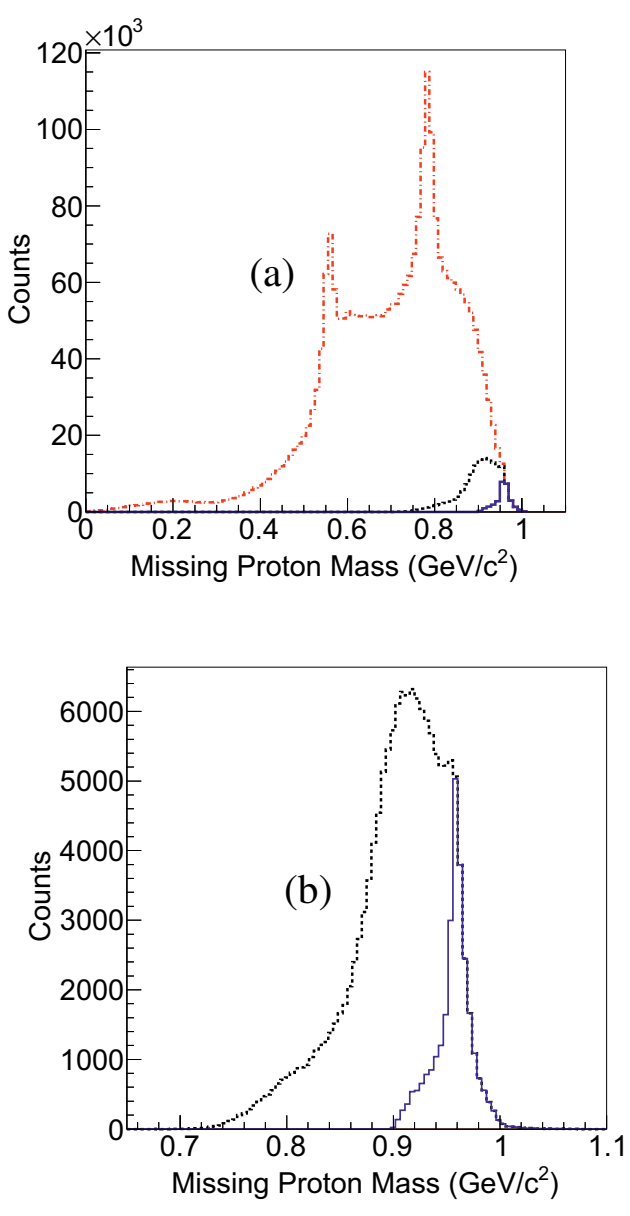

Figure 3. Panels a) and b) experimental proton missing mass with the cuts defined in condition i) (dash-dotted line), i) and ii) (dashel line) and i)-iii) (solid line).

In Fig. 3 panels a) and b) we present the missing mass of the detected proton, in the hypothesis of two particles in the reaction final state, by applying the condition i) (dash-dotted line), the conditions i) and ii) (dashed line), all conditions i-iii) (solid line). The figure clearly shows the identification of $\eta$ and $\omega$ mesons, while only after the application of the conditions i) and ii) the $\eta^{\prime}$ contribution starts to appear on the spectrum (see dotted line in figure). Finally, solid line in Fig. 3 (b) shows the $\eta^{\prime}$ signal contribution $\left(\eta^{\prime}\right.$ mass $\left.=0.95778 \mathrm{GeV}\right)$ dominant over a small and flat background.

The residual background was suppressed by additional constraints on the decay products of $\eta^{\prime}$ meson. In particular, we were able to identify the decay channels $\pi^{+} \pi^{-} \eta$ (B.R. 42.6\%), $\pi^{0} \pi^{0} \eta$ (B.R. 21.6\%) and $\gamma \gamma$ (B.R. $2.18 \%$ ).

The simplest and cleanest channel for the LAGRAN $\gamma \mathrm{E}$ detector is the $\eta^{\prime}$ decaying into $\gamma \gamma$. In this case, a similar analysis was used to that already used and published by the GRAAL collaboration in the cases of the $\pi^{0}$ and $\eta$ photoproduction off free proton and quasi-free proton (see Ref. [12, 14, 15, 17]). Unfortunately, the relatively low branching ratio does not allow us to collect enough events to extract a stable beam asymmetry. For this reason, a new analysis devoted to the identification of $\eta^{\prime}$ via
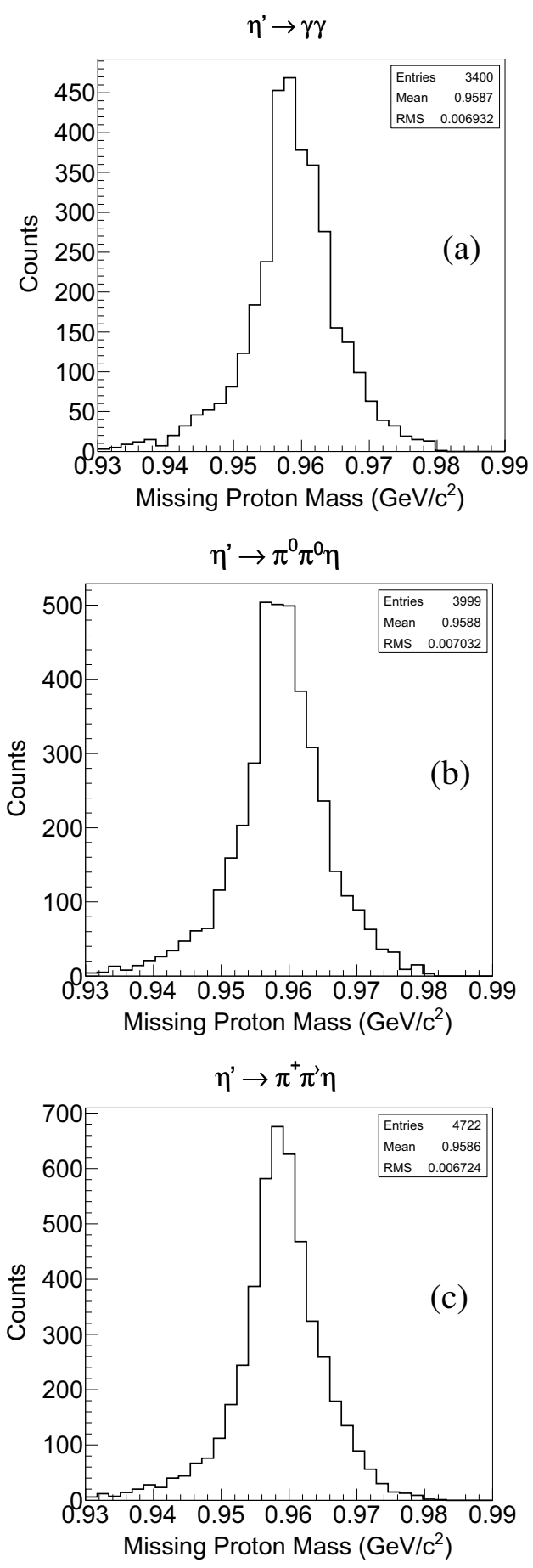

Figure 4. Proton missing mass for $\eta^{\prime} \rightarrow \gamma \gamma$ selection (panel a), $\eta^{\prime} \rightarrow \pi^{0} \pi^{0} \eta$ (panel b), and $\eta^{\prime} \rightarrow \pi^{+} \pi^{-} \eta$ (panel c).

its decay into $\pi^{0} \pi^{0} \eta$ and $\pi^{+} \pi^{-} \eta$ particles was implemented. The proton missing mass of selected events for $\gamma \gamma$ analysis is reported in Fig. 4 a).

The $\pi^{0} \pi^{0} \eta$ channel analysis was performed by asking to detect six neutral particles in the BGO, one proton in the forward direction and no other charged or neutral particle elsewhere. We reconstruct the invariant masses of $\eta^{\prime}$ from six photons and of $\eta$ and two $\pi^{0}$ decay products. The search of decay products was performed by looking to the best photon couple able to reconstruct the invariant mass of $\eta$ at first and using the remaining four photons for the re- 
construction of the invariant masses of the remaining two neutral pions. The proton missing mass of this selection is shown in in Fig. 4 b).

The charged decay channel was treated by applying the condition to have a good invariant mass reconstruction from two photons, detected in the BGO calorimeter, and the identification of two charged pions in the whole detector (see Fig. $4 \mathrm{c}$ )).

As one can see in Fig. 4, the missing mass of proton estimated for all analyzed decay modes are very similar in the different decay modes and very close to $\eta^{\prime}$ mass. By simulation we estimate that the residual background for the three considered cases is lower than $4 \%$.

The complete reconstruction of $\eta^{\prime}$ is not possible in the case of its decay into $\pi^{+} \pi^{-} \eta$ and has a bad resolution in the case of $\pi^{0} \pi^{0} \eta$ decay mode, while we are able to measure with good accuracy all quantities about the proton in the final state. For this reason, we derive all $\eta^{\prime}$ quantities in the center-of-mass frame from the well determined ones of the detected proton.
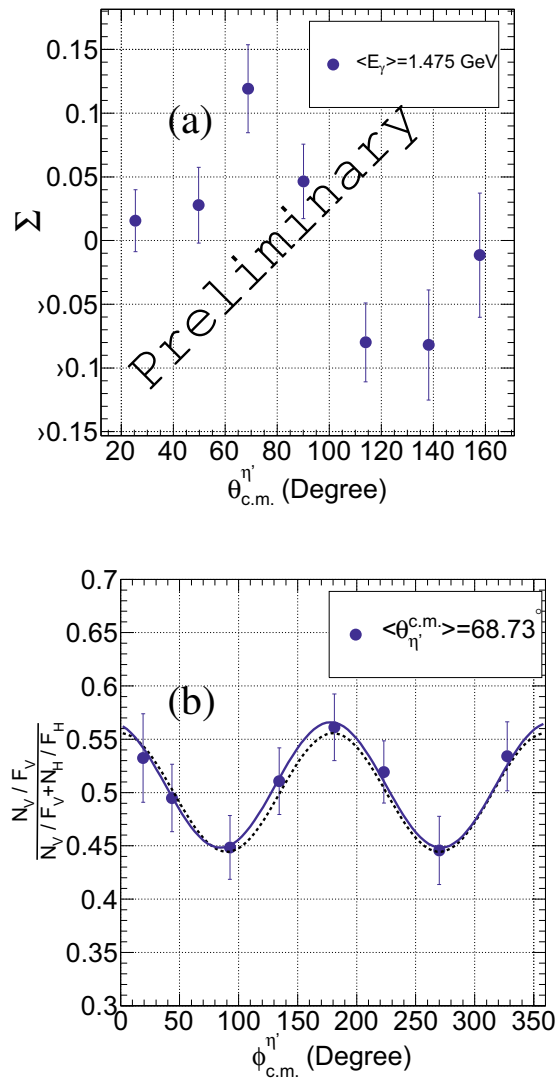

Figure 5. Preliminary $\eta^{\prime}$ beam asymmetries off proton at the average energy beam value of $1.475 \mathrm{GeV}$ (panel a). Azimuthal experimental distribution of the ratio $\left(N_{V} / F_{V}\right) /\left(N_{V} / F_{V}+N_{H} / F_{H}\right)$ (full circles), the fit of the ratio by eq. (1) by using $P \Sigma$ as only free parameter (dashed line), or by using a general parametrization of eq. (1) (solid line).

Fig. 5 shows the preliminary beam asymmetry of $\eta^{\prime}$ photoproduction off proton. In particular, we report in figure seven bins in $\theta_{c . m}^{\eta^{\prime}}$. estimated for the averaged en- ergy beam of about $1475 \mathrm{MeV}$. The beam asymmetry $\Sigma\left(E_{\gamma}, \theta_{c . m .}^{\eta^{\prime}}\right)$ was estimated at fixed intervals of $E_{\gamma}$ and $\theta_{c . m}^{\eta^{\prime}}$. by fitting the azimuthal distribution defined by the following ratio:

$$
\frac{N_{V} / F_{V}}{N_{V} / F_{V}+N_{H} / F_{H}}=\frac{1}{2}\left[1+P\left(E_{\gamma}\right) \Sigma \cos (2 \phi)\right]
$$

where the $N_{V}\left(N_{H}\right)$ and $F_{V}\left(F_{H}\right)$ are the number of events and the total flux for vertical (horizontal) beam polarization, while $P\left(E_{\gamma}\right)$ is the polarization degree of the beam calculated by QED [20]. The systematic error due to the efficiency $\epsilon$ is canceled in the ratio. The stability of the present results was verified by different checks (as in Ref. [15]).
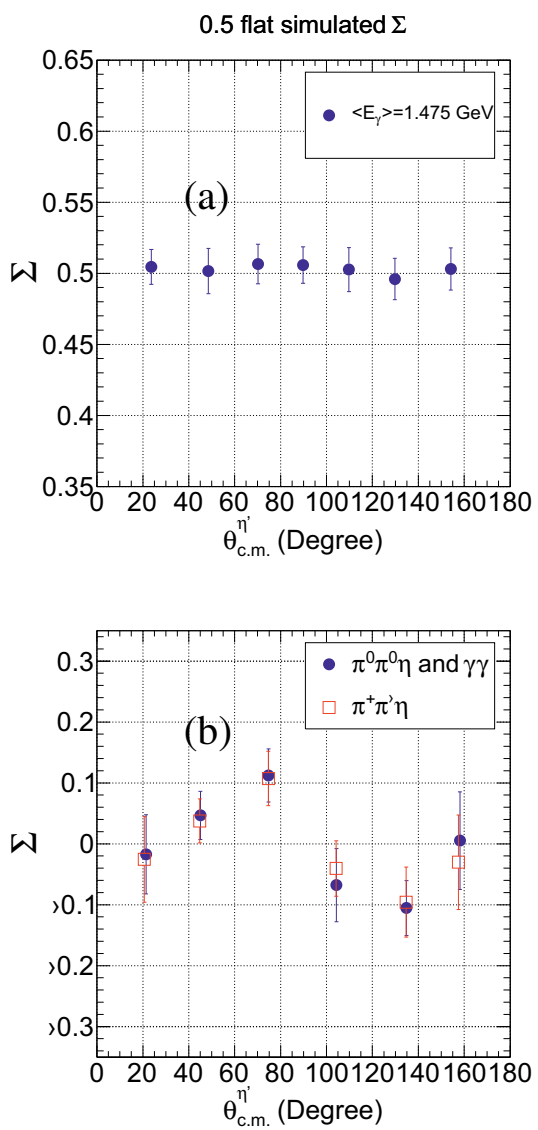

Figure 6. Beam asymmetry of a simulated sample generated with a 0.5 flat asymmetry (panel a). Beam asymmetries off proton obtained for the $\gamma \gamma$ and $\pi^{0} \pi^{0} \eta$ decay mode channels (full circles) and $\pi^{+} \pi^{+} \eta$ (empty squares) selection (panel b).

We fitted the azimuthal distributions by using different parametrizations of eq. (1). Parametrizing eq. (1) as $A[1+B \cos (C \phi+D)]$, we obtain that the quantities $A-0.5$, $C-2$ and $D$ are consistent with zero within the fitting error bar. In Fig. 5 b) we report the distribution of the ratio $\left(N_{V} / F_{V}\right) /\left(N_{V} / F_{V}+N_{H} / F_{H}\right)$ for $<E_{\gamma}>=1.475 \mathrm{GeV}$ and $<\theta_{c . m .}^{\eta^{\prime}}>=68.73^{\circ}$ (full circles) and the fit of the distribution by using $P\left(E_{\gamma}\right) \Sigma$ as only parameter (dotted line) and the general parametrization above described (solid line). The $P\left(E_{\gamma}\right) \Sigma$ parameters extracted in the two fits are in agreement within the error bars. 
We extract the beam asymmetry of a simulated sample generated with a 0.5 flat asymmetry. As one can see in Fig.6 a) the extracted asymmetries are compatible with the simulated ones within one standard deviation.

Fig. 6 b) shows the comparison between the beam asymmetry evaluated in the set of data obtained by looking for the $\pi^{+} \pi^{-} \eta$ decay and the one obtained with the sum of $\gamma \gamma$ and $\pi^{0} \pi^{0} \eta$ decay channels. The two estimations are in agreement within the error bars.

The trend of the beam asymmetry is in average zero confirming the dominant role of the s-wave function $[8,9]$, but the presence of a value clearly higher than zero for $\theta_{c . m}^{\eta^{\prime}}$. lower than $90^{\circ}$ hints the possible interference of different resonances.

\section{References}

[1] M. Benmerrouche, N.C. Mukhopadhyay and J.F. Zhang, Phys. Rev. D 51, 3237 (1995).

[2] B. Saghai and F. Tabakin, Phys. Rev. C 55, 917 (1997).

[3] D. Drechsel, O. Hanstein, S.S. Kamalov and L. Tiator, Nucl. Phys. A 645, 145 (1999).

[4] T. Feuster and U. Mosel, Phys. Rev. C 59, 460 (1999).

[5] W.-T. Chiang and F. Tabakin, Phys. Rev. C 55, 2034 (1997).
[6] F. Huang, H. Haberzettl, and K. Nakayama, Phys. Rev C 87, 054004 (2013).

[7] M. Dugger et al., Phys. Rev. Lett. 96, 062001 (2006).

[8] M. Williams et al., Phys. Rev. C 80, 045213 (2009).

[9] V. Crede et al., Phys. Rev. C 80, 055202 (2009).

[10] K. Nakayama and H. Haberzettl, Phys. Rev C 73, 045211 (2006).

[11] Wen-Tai Chiang et al., Phys. Rev. C 68, 045202 (2003).

[12] O. Bartalini et al., Eur. Phys. J. A 26, 399 (2005).

[13] A. Lleres et al., Eur. Phys. J. A 31, 79 (2007).

[14] O. Bartalini et al., Eur. Phys. J. A 33, 169 (2007).

[15] A. Fantini et al., Phys. Rev. C 78, 015203 (2008).

[16] A. Lleres et al., Eur. Phys. J A 39, 149 (2009).

[17] R. Di Salvo et al, Eur. Phys. J. A 42, 151 (2009).

[18] L. Federici et al., Il Nuovo Cimento B 59, 247 (1980).

[19] R. Caloi et al., Lettere al Nuovo Cimento 27, 339 (1980).

[20] D. Babusci et al., Phys. Lett. B 3551 (1995).

[21] G. Mandaglio et al., Phys. Rev. C 82, 045209 (2010).

[22] V. Vegna et al., arXiv:1306.5943.

[23] P. Corvisiero et al., Nucl. Instr. \& Meth. A 346, 433 (1994). 